\title{
Responsive graphene oxide hydrogel microcarriers for controllable cell capture and release
}

\author{
Jie Wang ${ }^{1}$, Guopu Chen ${ }^{2}$, Ze Zhao ${ }^{1}$, Lingyu Sun ${ }^{1}$, Minhan Zou ${ }^{1}$, Jian'an Ren ${ }^{2}$ and Yuanjin Zhao ${ }^{1^{*}}$
}

\begin{abstract}
Cell microcarriers have emerged as a powerful cell culture platform in biomedical areas, but their functions are usually limited to simply capturing and proliferating cells, because of the simplicity of their components. Thus, in this study, we developed a new near-infrared (NIR) light-responsive graphene oxide (GO) hydrogel microcarrier system for controllable cell culture. The microcarriers were generated by using capillary microfluidics to emulsify the GO dispersed poly( $\mathrm{N}$-isopropylacrylamide) (pNIPAM) and gelatin methacrylate (GelMA) pre-gel solution. The composite GO hydrogel microcarriers exhibited photothermally responsive cell capture, as well as the capacity for proliferation and release due to the NIR absorption of GO, the thermally responsive shape transition of pNIPAM, and the high biocompatibility of GelMA. It was found that the NIR-responsive GO hydrogel microcarriers could prevent the cultured cells from being attacked by the immune system and promote the formation of tumor models in immunocompetent mice, which is desired for tumor and drug research. These features make the NIR-responsive GO hydrogel microcarriers excellent functional materials for different biomedical applications.
\end{abstract}

Keywords: microfluidics, graphene oxide, microcarrier, hydrogel, tumor

\section{INTRODUCTION}

Recently, strategies for the on-demand capture, proliferation, and release of cells in biomimetic three-dimensional (3D) environments have received increasing attention in fields such as cell biology, drug discovery and tissue engineering [1-8]. In particular, microcarriers have emerged as an optimized strategy based on novel 3D biomaterial scaffold platforms, which have advantages in terms of space saving, cost effectiveness, and lower time and labor requirements for cell culture [8-10]. Several approaches have been employed for engineering cell mi- crocarriers, including micromolding, electrojetting, photolithography and microfluidics [10-14]. Among them, microfluidic emulsified microcarriers are the most attractive due to the unprecedented control over their size, shape, and morphology [14-18]. And thus various biocompatible microcarriers have been generated for biomedical applications by employing different types of biopolymers in the microfluidic emulsion templates [1923]. However, despite their merits, most of the current microcarriers are only suitable for the capture and proliferation of cells because they comprise simple material composites; whereas the function of on-demand cell release is rarely reached, which restricts the further applications of microcarriers in cell transfer and reuse. Therefore, the development of functional microcarriers is still desired.

In this study, we developed new near-infrared (NIR) light-responsive graphene oxide (GO) hydrogel microcarriers for controllable cell capture and release, as illustrated in Scheme 1a. As an emerging multifunctional material with extraordinary physical and chemical properties, GO has been investigated extensively in many areas, including chemical, environmental, and energy fields [24-31]. In addition, with the integration of biocompatible hydrogels, the composite GO hydrogel materials can be obtained with new properties, including high hydrophilicity, large specific surface area, physical analogue of extracellular matrix, improved mechanical properties and cell compatibility [32-37]. These features facilitate numerous new applications of GO hydrogel materials in cell culture and other biomedical applications. However, most of the current GO hydrogels have bulk structures that limit their capabilities as effective biomaterial scaffolds for cell research; and the potential value of GO hydrogel microcarriers with responsiveness to various stimuli for the on-demand capture and release

\footnotetext{
${ }^{1}$ State Key Laboratory of Bioelectronics, School of Biological Science and Medical Engineering, Southeast University, Nanjing 210096, China

${ }^{2}$ Department of General Surgery, Jinling Hospital, Medical School of Nanjing University, Nanjing 210002, China

" Corresponding author (email: yjzhao@seu.edu.cn)
} 

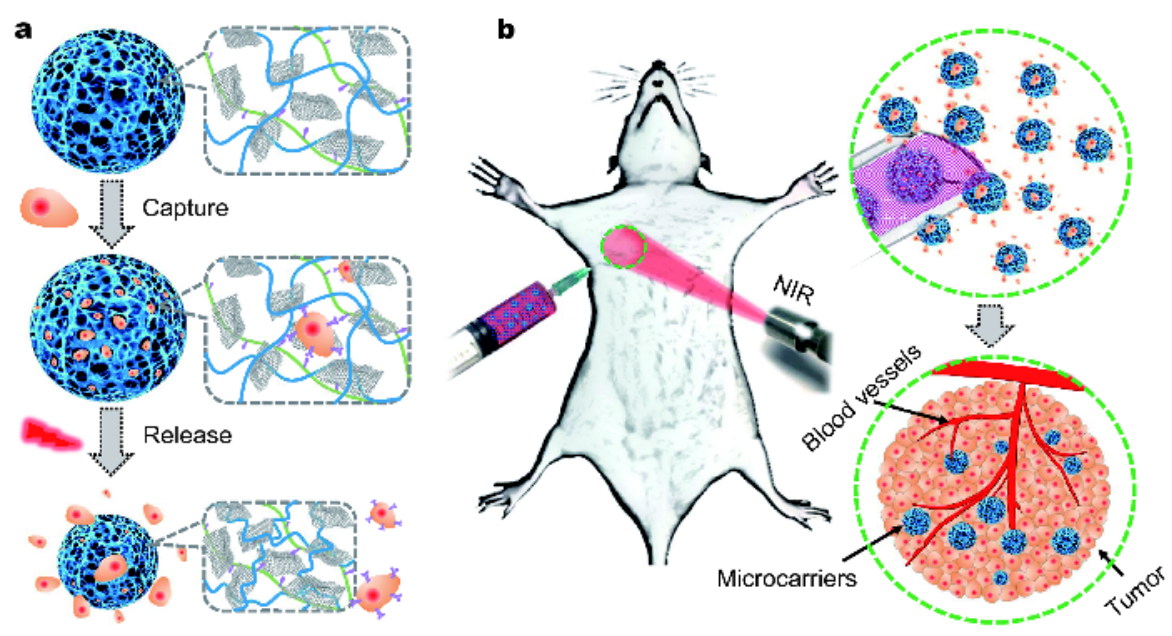

Scheme 1 (a) Schematic diagram of the NIR-responsive GO hydrogel microcarriers for controllable cell capture and release; (b) the responsive GO hydrogel microcarriers promote the formation of tumor models in immunocompetent mice with the NIR irradiation.

of cells has yet to be exploited.

Herein we employed a capillary microfluidic method to generate the desired GO microcarriers incorporated with poly ( $N$-isopropylacrylamide) (pNIPAM) and gelatin methacrylate (GelMA) hydrogels. The composite GO hydrogel microcarriers exhibited photothermally responsive cell capture, as well as the capacities for proliferation and release due to the NIR-absorption by GO, the thermally responsive shape transition of the PNIPAM, and the biocompatibility of GelMA. It was demonstrated that the responsive GO hydrogel microcarriers could prevent the cultured cells from being attackedby the immune system, and promote the formation of tumor models in immunocompetent mice (Scheme 1b). These features indicate that GO hydrogel microcarriers could be of great importance in biomedical research.

\section{EXPERIMENTAL SECTION}

\section{Materials}

$N$-isopropylacrylamide (NIPAM, 97\%), $N, N^{\prime}$-methylenebis(acrylamide) (BIS), and the photo-initiator 2-hydroxy2-methylpropiophenone (HMPP) were purchased from Sigma-Aldrich (USA). Acrylamide (AAm) was obtained from Aladdin Industrial Corporation (China). The graphene oxide (GO) solution was bought from Nanjing XF NANO Materials Tech Co., Ltd (China). Silicone oil (Shinetsu, 100cs, Japan) was used as the outer phase of the microfluidic device. Gelatin methacrylate (GelMA) was self-synthesized according to literature [38]. Briefly, methacrylic anhydride (MA) was added to $10 \%(w / v)$ gelatin (Type A, 300 bloom from porcine skin) in phos- phate buffered saline (PBS) solution. After reaction, the solution was dialyzed to remove salts and methacrylic acid and freeze-dried before use. MA, gelatin, and PBS were bought from Sigma-Aldrich (USA). Water with a resistivity of $18.2 \mathrm{M} \Omega \mathrm{cm}^{-1}$ was acquired from a Millipore Milli-Q system. All other chemical reagents were of the best grade available and used as received.

\section{Microfluidics}

The capillary microfluidic device consists of coaxial assemblies of two (inner and outer) round and a square glass capillaries on glass slides. To construct the microfluidic device for fabricating single emulsions, the inner capillary (with an inner diameter of $0.8 \mathrm{~mm}$ and an outer diameter of $1 \mathrm{~mm}$ ) was prepared by using a capillary puller (P-97, Sutter Instrument, Novato, CA, USA) and a microforge (MF-830, Narishige, Tokyo, Japan) having a tapered tip with an inner diameter of $0.1-0.3 \mathrm{~mm}$, and was treated with $5 \mathrm{vol} \%$ ethanol solution of 3 -aminopropyl triethylene silane (APTES, Alfa Aesar, UK) for over $24 \mathrm{~h}$ to aminate the surface before use. The collection (outer) capillary tube (with an inner diameter of $0.8 \mathrm{~mm}$ and an outer diameter of $1 \mathrm{~mm}$ ) was dispersed in a hydrophobic reagent (trimethoxy (octadecyl) silane, Sigma-Aldrich Co., USA). These inner and outer capillaries were coaxially assembled in a square capillary with an inner diameter of $1.05 \mathrm{~mm}$ (AIT Glass, Rockaway, NJ, USA). A transparent epoxy resin was used to seal the tubes where required. To generate the single emulsion templates, all fluids were pumped into the capillary microfluidic device using syringe pumps (PHD 2000 series, Harvard, Plymouth Meeting, PA, USA). The generated 
emulsions were polymerized in the collection channel with an ultraviolet light $(365 \mathrm{~nm}$, S1000, OmniCure, Mississauga, Ontario, Canada) and then were collected in glass vials that were filled with deionized water. The collected microcarriers were washed to remove the outer phase and freeze-dried.

\section{Generation of jellyfish-shaped GO hydrogels and GO hydrogel microcarriers}

For a typical experiment, $10 \mathrm{wt} \%$ NIPAM, $0.34 \mathrm{wt} \% \mathrm{BIS}$, 2.5 wt $\%$ GelMA, $0.2 \mathrm{wt} \%$ GO, and 1 vol\% HMPP were dispersed in water homogeneously and polymerized under UV light at a distance of $5 \mathrm{~cm}$ to $10 \mathrm{~cm}$ for $5-20 \mathrm{~s}$ with a jellyfish-shaped mask or in the microfluidics device, to fabricate jellyfish-shaped GO hydrogels or GO hydrogel microcarriers, respectively. For investigating the lower critical solution temperature (LCST) of the GO hydrogel, the total concentration of NIPAM and AAm was kept at $10 \mathrm{wt} \%$, with $0.34 \mathrm{wt} \%$ BIS, $2.5 \mathrm{wt} \%$ GelMA, $0.2 \mathrm{wt} \% \mathrm{GO}$, and $1 \mathrm{vol} \%$ HMPP. The volume ratio versus different temperature was recorded precisely by keeping the corresponding temperature for $5 \mathrm{~min}$ to make the volume change stable.

\section{Cell loading and culture}

HepG2 cells and Hepa1-6 cells (obtained from the Cell Bank of the Chinese Academy of Sciences, Shanghai, China) were incubated with sufficient culture medium in the incubator (HERA cell 150, Thermo, USA) with 5\% $\mathrm{CO}_{2}$ at $37^{\circ} \mathrm{C}$. The culture medium was a high sugar Dulbecco's modified Eagle's medium (High Glucose DMEM, Invitrogen, Nanjing and Biotech Development Co. Ltd) supplemented with $10 \%$ fetal bovine serum (FBS, Gibco), $1 \% \quad 10,000$ units $\mathrm{mL}^{-1}$ penicillin and $10,000 \mu \mathrm{g} \mathrm{mL}^{-1}$ streptomycin (Gibco, Life Technolegy). The freeze-dried GO hydrogel microcarriers were infused with cell suspensions to load cells. The quantities of cells released by near-infrared irradiation (NIR, $808 \mathrm{~nm}, \mathrm{Xi}$ Long Tech Co., Ltd, China) and loaded on microcarriers were countered by Handheld Automated Cell Counter (Scepter ${ }^{\mathrm{TM}}$, Millipore, USA). The viabilities were tested $2 \mathrm{~h}$ after the cell release before the proliferation in live/dead assays. During the assays, the cells were stained by $10 \mu \mathrm{mol} \mathrm{L}{ }^{-1}$ calcein-AM (Molecular Probes Co.) and propidium iodide (PI) (Molecular Probes Co.) for observation. In the NIR-stimulated cell release experiment, the microcarrier was irradiated at $0.5 \mathrm{~W} \mathrm{~cm}$.2 NIR at around $15 \mathrm{~s}$ per cycle for 12 cycles. 3-(4,5-Dimethylthiazol-2-yl)-2,5-diphenyltetrazolium bromide (MTT) (98\%, J\&K Scientific Ltd., Shanghai) was used for quantitative analysis of cell viability. The number of replicates was 8 .

\section{Mice tumor models}

The 6-8 weeks, $18-25 \mathrm{~g}$, male C57BL/6J mice were supplied by Comparative Medicine of Jinling Hospital (Nanjing, China). Animals were treated in strict accordance with the recommendations in the Guide for the Care and Use of Laboratory Animals of the National Institutes of Health (USA). All the animal care and experimental protocols were reviewed and approved by the Animal Investigation Ethics Committee of Jinling Hospital. The mice were randomly divided into three groups: NIR (-) Cells group (Group 1), NIR (-) Microcarriers group (Group 2), and NIR (+) Microcarriers group (Group 3) with ten mice in each group. Each mouse was anaesthetic by 5 vol\% $10 \mathrm{mg} \mathrm{mL}^{-1}$ chloral hydrate before injection.

Hepa1-6 cells and microcarriers loaded with Hepa1-6 cells were dispersed in DMEM without FBS for injection subcutaneously to the armpit regions of mice. The numbers of the injected cells $\left(2 \times 10^{5}\right.$ cells of each mouse) were kept the same for all the mice in each group. The mice were then put back to cages and allowed free access to water and food. Mice in Group 3 were depilated and then exposed to NIR with the power intensity of $0.5 \mathrm{~W} \mathrm{~cm}^{-2}$ for about $20 \mathrm{~s}$ in the second day after injection. The irradiation continued for 4 days with the frequency of one time a day. 14 days later, the mice were sacrificed to extract the tumors for investigation.

\section{Characterization}

The microcarrier generating processes in the capillary microfluidic device were monitored using a microscope (SMZ 745T, Nikon, Tianjin, China) fitted with a fast camera (S-PRI F1, AOS Technologies AG). Optical microscopy images of microcarriers were obtained with a stereo microscope (MVX10, Olympus, Tokyo, Japan) and recorded by a charged coupled device (CCD, Media Cybernetics Evolution MP 5.0 RTV). Fluorescence images of the microcarriers were obtained by an inverted fluorescence microscope (IX71, Olympus, Tokyo, Japan) equipped with CCD cameras (DP30BW, Olympus, Tokyo, Japan). Morphology features of the microcarriers were explored by the scanning electron microscopy (SEM) images using a field emission scanning electron microscope (FESEM, Ultra Plus, Zeiss). The temperature of the mice was recorded by the uncooled handheld infrared camera (FLIR Systema AB, Sweden) with a spotmeter automatically focused on the highest temperature site in sight. The tumors in Group 2 and 3 were weighted 
$\mathbf{a}$

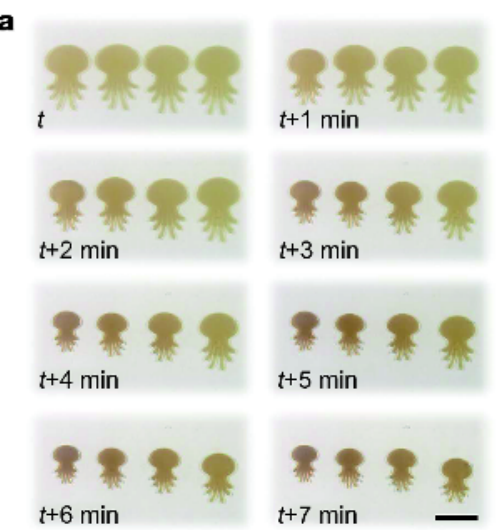

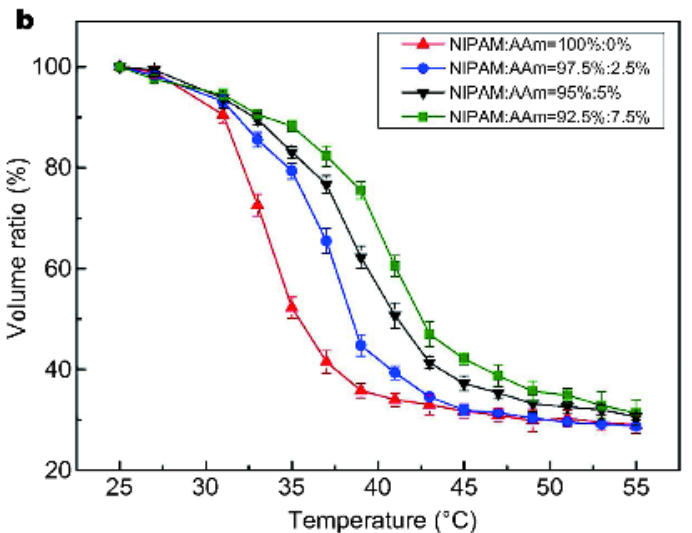

Figure 1 (a) The thermoresponsive behaviors of the jellyfish-shaped GO composite hydrogels with the temperature increasing. The concentration ratios of NIPAM:AAm in the composite hydrogels were 100\%:0\%, 97.5\%:2.5\%, 95\%:5\%, and 92.5\%:7.5\% from left to right, respectively. The scale bar is $5 \mathrm{~mm}$. (b) The volume ratios of the corresponding composite hydrogels in (a) at different temperature.

after eliminating the microcarriers. For histopathological characterization, the tumors were dipped in formaldehyde, followed by dehydration and then embedded in paraffin. Serially section was obtained by a microtome according to standard protocols. The sections, $5 \mu \mathrm{m}$ in thickness, were prepared for hematoxylin and eosin staining (H\&E) and immunohistochemical evaluation with CD31, Ki67.

\section{RESULTS AND DISCUSSION}

In a typical experiment, the stimuli-responsive behaviors of the GO composite hydrogel materials were firstly investigated and optimized. GO has been found to possess an extraordinary capacity for photothermal energy transformation due to the strong optical absorption of NIR, and the pNIPAM hydrogel exhibits a thermoresponsive property [39]. Therefore, NIR-responsive phase transition performance was expected for the combination of GO and pNIPAM composite hydrogel. It is well known that the pNIPAM hydrogel exhibits a sharp transition from a hydrophilic swollen state to a hydrophobic collapsed state when the temperature exceeds its LCST of around $32^{\circ} \mathrm{C}$. While a suitable LCST for a composite hydrogel between body temperature $\left(37^{\circ} \mathrm{C}\right)$ and hyperthermia temperature $\left(42^{\circ} \mathrm{C}\right)$ is desirable for in vivo cell experiments. And thus, AAm was introduced to increase the LCST, as shown in Fig. 1. For better immobilization and more intuitive volume change, the jellyfish-shaped composite hydrogel was fabricated to illustrate the thermoresponsive behaviors of the composite GO hydrogel. It was found that as the concentration ratio of AAm:NIPAM increased, the LCST of the composite GO hydrogel increased gradually, and an opti- mized LCST of around $39^{\circ} \mathrm{C}$ was achieved when the concentration ratio of NIPAM:AAm was $92.5 \%: 7.5 \%$. The GelMA solution was also incorporated for copolymerization to increase the biocompatibility and cell-adhesive properties of the composite hydrogel. The concentration of both GelMA and NIPAM could influence the volume ratios of the composite hydrogel, as shown in Fig. S1, which indicates that the responsiveness of the composite hydrogel decreased as the concentrations of NIPAM or GelMA increased, thereby decreasing the cell release efficiency. Comprehensively considering the mechanical stability and the cell release efficiency of the composite hydrogel, the concentration ranges of $5-20 \mathrm{wt} \%$ for NIPAM and $0.5-4.5$ wt $\%$ for GelMA was employed in our subsequent experiments. Under these concentrations of the components, the GO hydrogel exhibited obvious volume responsiveness under NIR irradiation, which was confirmed by SEM in Fig. S2.

In order to fabricate the responsive GO hydrogel microcarriers, a simple capillary microfluidic device assembled via the coaxial alignment of two cylindrical capillaries was used to generate water in oil single emulsion templates, as shown in Fig. 2a and Fig. S3a. The aqueous GO composite pre-gel solution was pumped as the dispersed inner phase, while silicone oil was driven into the microfluidic device as the continuous outer phase from the same direction. The GO composite pre-gel solution was emulsified continuously into droplets and subsequently polymerized by ultraviolet irradiation. Microcarriers with high monodispersity were obtained using this microfluidic method, where the overall sizes of the microcarriers could be controlled by adjusting the flow rates of the inner and outer phases, as shown in Fig. S3. 

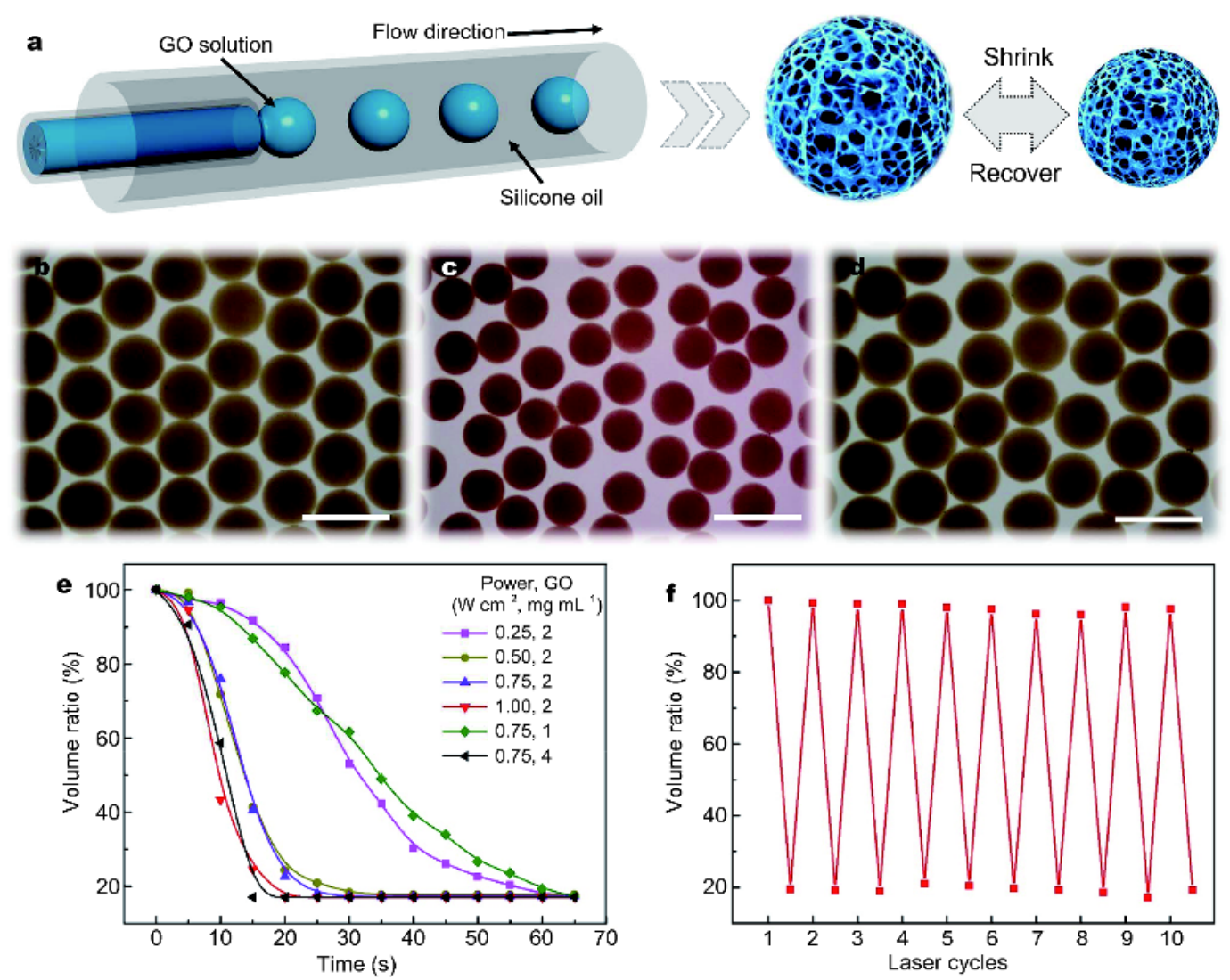

Figure 2 (a) Schematic diagram of microfluidic generation of responsive GO hydrogel microcarriers. (b-d) The optical microscopic images of the GO hydrogel microcarriers before, under, and after NIR irradiation, respectively. The scale bars are $1 \mathrm{~mm}$. (e) The volume change of GO hydrogel microcarriers with different concentration of GO under different power intensity of NIR. (f) The volume variation of GO hydrogel microcarriers as a function of the cycle number.

The GO hydrogel microcarriers obtained were also responsive to NIR irradiation due to their photothermal GO and thermoresponsive pNIPAM components. The NIR-responsive behaviors of the GO hydrogel microcarriers were investigated by optical characterization in Fig. 2b-d. It was found that under NIR irradiation, the GO hydrogel microcarriers exhibited maximum volume shrinkage to approximately $20 \%$ of their original size. The GO hydrogel microcarriers returned to their original extended state when the NIR laser was switched off. The NIR-responsive efficiency of the microcarriers was further evaluated as the functions of the NIR laser power intensity and the concentration of the GO solution, as shown in Fig. 2e. It was found that the rate of the decrease in the volume increased as the NIR laser power intensity or GO concentration increased within a certain range. The rate of the decrease in the volume increased no longer obviously when the GO concentration exceeded $2 \mathrm{mg} \mathrm{mL}$. Thus, an optimized GO concentration of
$2 \mathrm{mg} \mathrm{mL}^{-1}$ was chosen. Under these optimized conditions, reversible changes in the volume ratios of the microcarriers were also investigated, as shown in Fig. 2f. The results indicated that the microcarriers had good durability over several cycles, and their responsiveness was repeatable and reversible. It should be noted that compared with other stimuli, the intensity and wavelength of NIR light can be controlled easily and remotely as a rapid and precise trigger; and NIR has been extensively considered as a tissue transparency window due to its capacity for penetrating human tissues well without causing harm. Thus, the GO hydrogel microcarriers may have potential uses in in vivo experiments.

The cell capture and on-demand release capacities of the microcarriers were evaluated to confirm the biomedical value of the GO hydrogel microcarriers. First, the biocompatibility of the microcarriers was evaluated using the MTT assay, which is the most common method for the quantitative investigation of cell viability, as shown in 

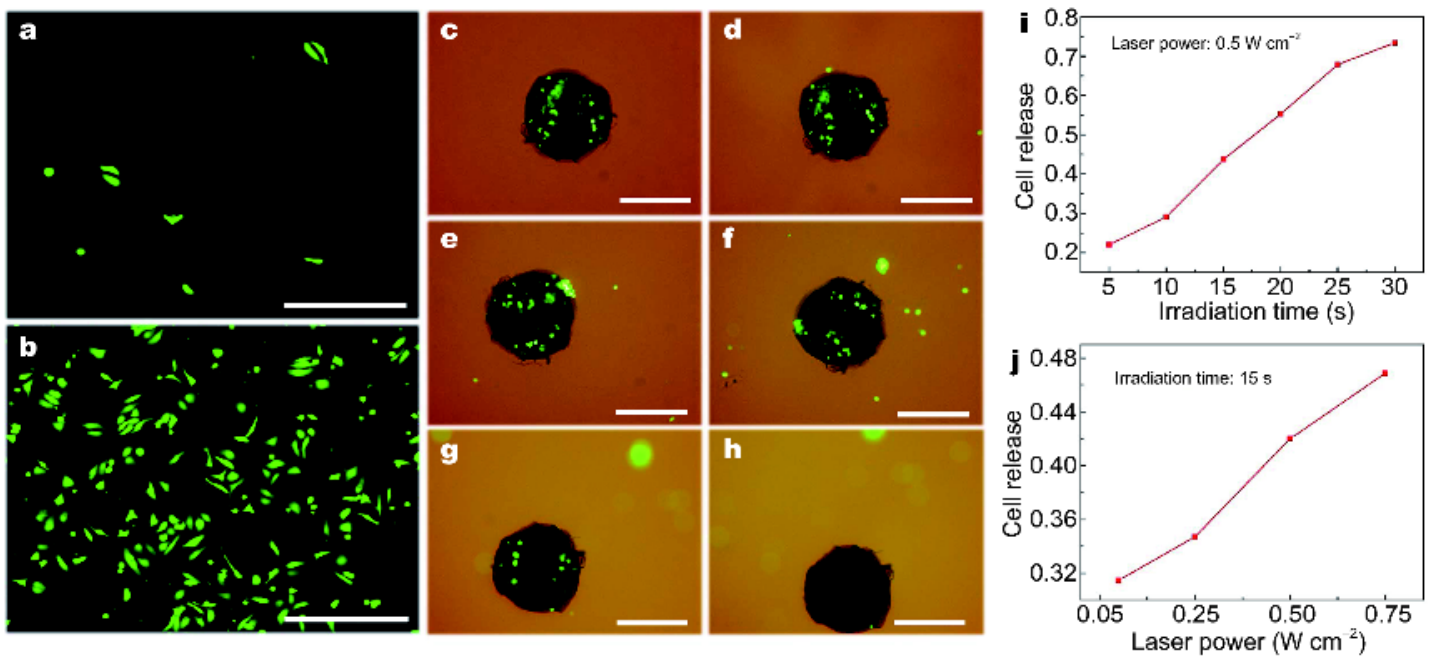

Figure 3 (a, b) Fluorescent images of (a) escaped cells and (b) released cells from the microcarriers. (c-h) The progress of NIR-stimulated cell release from the microcarrier during several cycles. (i, j) The percentage of released cells with different irradiation period and laser power intensity, respectively. The scale bars are $150 \mu \mathrm{m}$ in $(\mathrm{a}, \mathrm{b})$ and $500 \mu \mathrm{m}$ in $(\mathrm{c}-\mathrm{h})$.

Fig. S4. It was found that the incorporation of GelMA into the microcarriers clearly increased the cell viability, whereas the cell viabilities in microcarriers with different concentrations of GelMA ranging from 0.5 to $4.5 \mathrm{wt} \%$ did not differ greatly. An average GelMA concentration of $2.5 \mathrm{wt} \%$ was employed in the cell studies. To investigate the capture capacity of the microcarriers, HepG2 cells were used as the model cells, which were much smaller than the pores of the GO hydrogel materials. Freeze-dried GO hydrogel microcarriers were infused with cell suspensions to load the cells. The surrounding cell suspensions were adsorbed gradually into the microcarriers and only a negligible number of cells (less than $8 \%$ ) escaped from the microcarriers (Fig. 3a, b). The captured cells were unlikely to fall off the GO hydrogel microcarriers during their conventional incubation, which may be ascribed to the presence of the GelMA hydrogel providing numerous molecular sites for cell attachment.

To verify the cell release capacity of the GO hydrogel microcarriers, the process of NIR-stimulated cell release was recorded in Fig. 3c-h. The results indicated that after exposure to NIR irradiation, the cell microcarriers shrank rapidly and expelled the cells together with the culture medium. The microcarriers recovered their original shape within a few seconds after the NIR was switched off. All of the cells were released eventually after several cycles because of the repeatable and reversible responsiveness of the microcarriers. The release rates of the microcarriers were further investigated using different NIR laser power intensities and irradiation periods (Fig. 3i, j), which showed that the release rate increased as the irradiation period and laser power intensity increased. And thus, the cell release rate and dose of the GO hydrogel microcarriers were easy to control.

Considering the need for cell reuse, the viability of the released cells is critical and the efficient release of cells with minimum damage is desirable. A series of laser power intensities with a fixed irradiation time were tested in order to optimize the irradiation conditions by evaluating the viability of the released cells by staining in living/dead assays. As shown in Fig. S5, more cells were released as the irradiation power intensity increased, but many more dead cells emerged when the power intensity exceeded $0.75 \mathrm{~W} \mathrm{~cm}^{-2}$. After considering the release efficiency and damage during release, a power intensity of $0.5 \mathrm{~W} \mathrm{~cm}^{-2}$ was employed for release. In addition, the irradiation period should be less than $15 \mathrm{~s}$ in order to avoid overheating the cell medium above the hyperthermia temperature of $42^{\circ} \mathrm{C}$ (Fig. S6). Under these optimized conditions, MTT assays were conducted to further demonstrate the viability of the released cells, which were also compared with enzyme-digested cells, as shown in Fig. S7. The results indicated that both methods obtained similar cell viability levels over 3 days, which suggested that the proposed release approach using NIR had little effect on the proliferation of cells. Therefore, the GO hydrogel microcarriers provide a facile and biologically friendly platform for controllable cell capture and release.

To demonstrate the value of the GO hydrogel micro- 


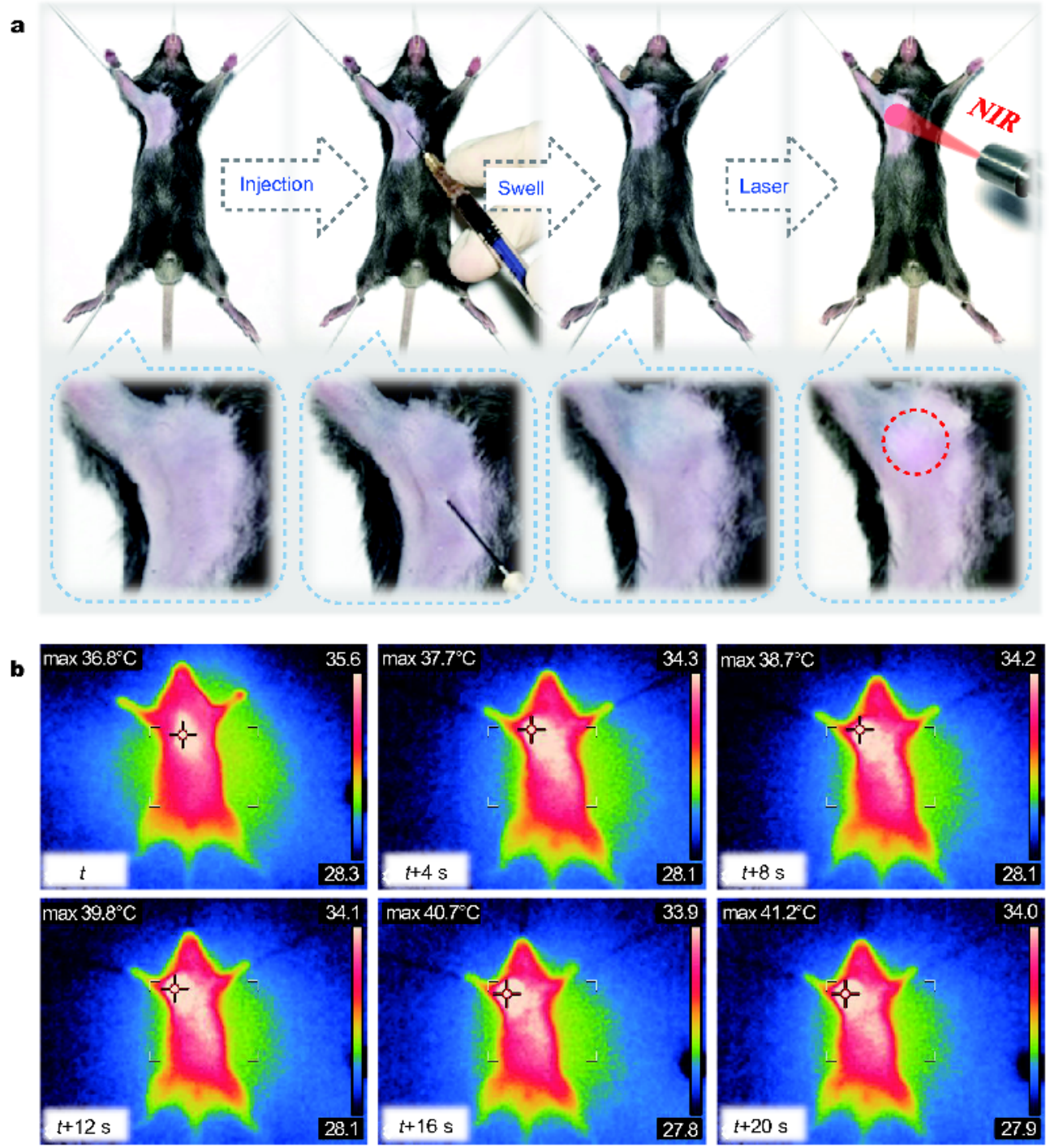

Figure 4 (a) The progress of injecting microcarriers loaded with Hepa1-6 cells into the armpits of mice and releasing cells subsequently by NIR. (b) The progress of the temperature change of the model under NIR irradiation.

carriers for in vivo use in experiments, they were employed for constructing tumor models in immunocompetent mice. Most tumor models were built in immunodeficient mice due to the high tumor formation rate but the immune system had a great effect on the tumor microenvironment in vivo, which should not be ignored when studying tumors. Thus, the construction of tumor models in immunocompetent mice is important for many practical studies. Hepal-6 cells loaded GO hydrogel microcarriers were injected subcutaneously into the armpit regions of mice and subsequently irradiated by NIR to release the cells, as shown in Fig. 4. After injection, it could be observed that the black microcarriers were still visible below the skin of the mouse, which allowed accurate NIR irradiation at the site. The capacity of the NIR laser to penetrate the mouse skin was investigated, as shown in Fig. S6. It was found that the NIR-driving photothermal effect of the microcarriers could still be induced with little degradation in vivo, which could be compensated by increasing the irradiation time or the laser power intensity in a convenient manner. The NIRstimulated responsiveness of the microcarriers was investigated by recording the temperature of the model under NIR, as shown in Fig. $4 \mathrm{~b}$. The results showed that the highest temperature point in the mouse changed from the heart to the microcarrier-loaded site immediately 

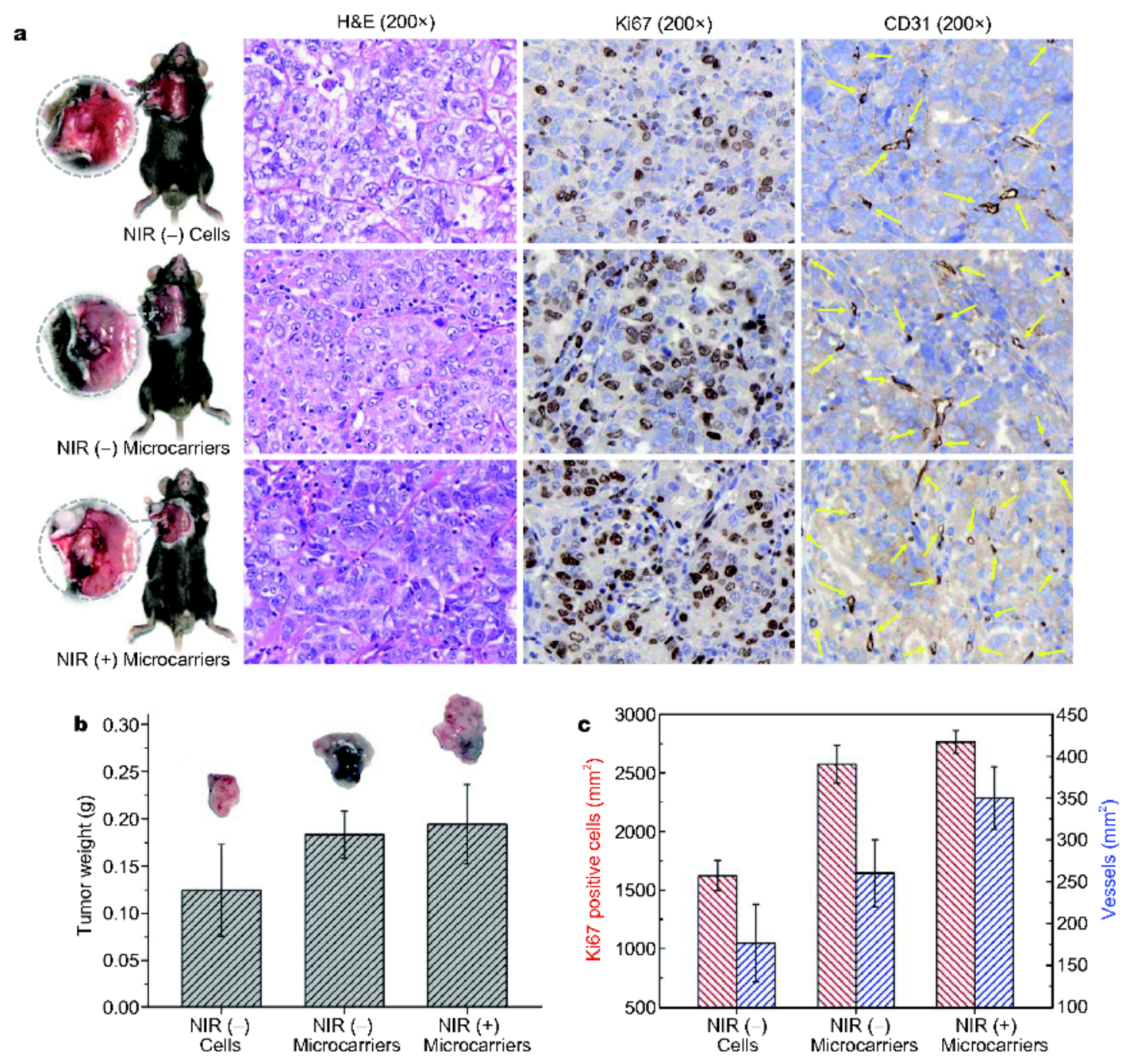

Figure 5 (a) The comparison of the tumor proliferation and angiogenesis in three groups, determined by H\&E, Ki67 and CD31 staining. The vessels are pointed with the yellow arrows. (b) Quantification of the tumor weight in three groups. The colors of the tumors are not uniform for different mice due to the random encapsulation of the black microcarriers in the tumors. (c) Quantification of Ki67 and CD31 staining shown by the positively stained cells density and blood vessel density, respectively. Results are presented as mean value \pm standard error in (b) and (c).

after NIR irradiation, where the temperature of the microcarriers increased above the LCST within a few seconds. These indicated that the GO hydrogel microcarriers also exhibit good responsiveness and a high capacity for cell release in vivo.

In order to further confirm the suitability of the cellloaded microcarriers for building tumor models in vivo, three mixtures (pure cell suspension, cell-loaded microcarriers without NIR irradiation, and cell-loaded microcarriers with periodic NIR irradiation) containing the same quantities of cells were injected into the immunocompetent mice, as shown in Fig. 5. The results showed that the tumors developed in all three groups, with the tumor formation rates of around 60\%-70\%, which did not show much increase in the GO hydrogel microcarrier groups. However, the tumors in the direct cell injection group were smaller than those in the GO hydrogel microcarrier groups regardless of whether the NIR was switched on or off (Fig. 5b). This should be ascribed to the capacity of the microcarriers for protecting the cells. In general, cells tend to undergo excessive shear forces and risk of leakage when they are injected directly without extra protection. Thus, the protection provided by the microcarriers could decrease the mechanical damage caused during injection, prevent attack by the immune system, and thereby facilitate in vivo cell 
retention, engraftment, survival, and ultimate tumor formation.

Tumor proliferation and angiogenesis were also investigated by histopathological characterization, as shown in Fig. 5a, c. The results demonstrated that the tumors from the microcarrier groups had higher proliferation rates (determined by $\mathrm{Ki} 67$ labeling and $\mathrm{H} \& \mathrm{E}$ staining) and vessel numbers (determined by CD31 labeling), thereby indicating that the tumor proliferation and angiogenesis were facilitated by the microcarriers. In particular, there were more vessels in the tumors in the group with the microcarriers subjected to NIR irradiation than in the microcarrier group without irradiation. This may be explained by the repeated swelling and deswelling effects of the microcarriers under periodic NIR irradiation, which could effectively accelerate tumor angiogenesis. These results indicate that the responsive GO hydrogel microcarriers provide an efficient platform for building tumor models with facilitated tumor proliferation and angiogenesis in immunocompetent mice, which is desired for tumor and drug research.

\section{CONCLUSIONS}

In summary, we successfully developed new NIR-responsive microcarriers with GO, pNIPAM, and GelMA components for controllable cell capture and release. The overall sizes, monodispersity, and the concentrations of the different components of the microcarriers were well tailored using a droplet microfluidic method. The photothermal effect of GO under NIR, the thermoresponsiveness of pNIPAM, and the biocompatibility of GelMA, provided the GO hydrogel microcarriers with capacities for the on-demand capture, proliferation, and release of cells. With optimal GO/hydrogel concentrations and irradiation conditions, cells with high viability could be released effectively from the microcarriers, which is very important for cell transfer and reuse. We also demonstrated the value of the NIR-responsive GO hydrogel microcarriers for facilitating tumor proliferation and angiogenesis in immunocompetent mice models. These features indicate that the NIR-responsive GO hydrogel microcarriers could have numerous biological and biomedical applications.

Received 3 February 2018; accepted 14 March 2018; published online 12 April 2018

1 Wang L, Liu H, Zhang F, et al. Smart thin hydrogel coatings harnessing hydrophobicity and topography to capture and release cancer cells. Small, 2016, 12: 4697-4701

2 Yuan B, Jin Y, Sun Y, et al. A strategy for depositing different types of cells in three dimensions to mimic tubular structures in tissues. Adv Mater, 2012, 24: 890-896

3 Li Y, Liu W, Liu F, et al. Primed 3D injectable microniches enabling low-dosage cell therapy for critical limb ischemia. Proc Natl Acad Sci USA, 2014, 111: 13511-13516

$4 \mathrm{Hu} \mathrm{K}$, Zhou N, Li Y, et al. Sliced magnetic polyacrylamide hydrogel with cell-adhesive microarray interface: a novel multicellular spheroid culturing platform. ACS Appl Mater Interfaces, 2016, 8: 15113-15119

5 Frey O, Misun PM, Fluri DA, et al. Reconfigurable microfluidic hanging drop network for multi-tissue interaction and analysis. Nat Commun, 2014, 5: 4250

6 Wang W, Cui H, Zhang P, et al. Efficient capture of cancer cells by their replicated surfaces reveals multiscale topographic interactions coupled with molecular recognition. ACS Appl Mater Interfaces, 2017, 9: 10537-10543

7 Huang C, Yang G, Ha Q, et al. Multifunctional "smart" particles engineered from live immunocytes: toward capture and release of cancer cells. Adv Mater, 2015, 27: 310-313

8 Asghar W, El Assal R, Shafiee H, et al. Engineering cancer microenvironments for in vitro 3-D tumor models. Mater Today, 2015, 18: 539-553

9 Liu X, Jin X, Ma PX. Nanofibrous hollow microspheres self-assembled from star-shaped polymers as injectable cell carriers for knee repair. Nat Mater, 2011, 10: 398-406

10 Liu Z, Shum HC. Fabrication of uniform multi-compartment particles using microfludic electrospray technology for cell coculture studies. Biomicrofluidics, 2013, 7: 044117

11 Chan HF, Zhang Y, Leong KW. Efficient one-step production of microencapsulated hepatocyte spheroids with enhanced functions. Small, 2016, 12: 2720-2730

12 Tang MYH, Shum HC. One-step immunoassay of C-reactive protein using droplet microfluidics. Lab Chip, 2016, 16: 4359-4365

13 Griffin DR, Weaver WM, Scumpia PO, et al. Accelerated wound healing by injectable microporous gel scaffolds assembled from annealed building blocks. Nat Mater, 2015, 14: 737-744

14 Shang L, Cheng Y, Zhao Y. Emerging droplet microfluidics. Chem Rev, 2017, 117: 7964-8040

15 Sun J, Zhang L, Wang J, et al. Tunable rigidity of (polymeric core)(lipid shell) nanoparticles for regulated cellular uptake. Adv Mater, 2015, 27: 1402-1407

16 Sim JY, Lee GH, Kim SH. Microfluidic design of magnetoresponsive photonic microcylinders with multicompartments. Small, 2015, 11: 4938-4945

17 Lee SS, Kim B, Kim SK, et al. Robust microfluidic encapsulation of cholesteric liquid crystals toward photonic ink capsules. Adv Mater, 2015, 27: 627-633

$18 \mathrm{Yu} \mathrm{Y,} \mathrm{Fu} \mathrm{F,} \mathrm{Shang} \mathrm{L,} \mathrm{et} \mathrm{al.} \mathrm{Bioinspired} \mathrm{helical} \mathrm{microfibers} \mathrm{from}$ microfluidics. Adv Mater, 2017, 29: 1605765

19 Liu W, Shang L, Zheng F, et al. Photonic crystal encoded microcarriers for biomaterial evaluation. Small, 2014, 10: 88-93

20 Zhao Y, Shang L, Cheng Y, et al. Spherical colloidal photonic crystals. Acc Chem Res, 2014, 47: 3632-3642

21 Wang J, Cheng Y, Yu Y, et al. Microfluidic generation of porous microcarriers for three-dimensional cell culture. ACS Appl Mater Interfaces, 2015, 7: 27035-27039

22 Fu F, Shang L, Zheng F, et al. Cells cultured on core-shell photonic crystal barcodes for drug screening. ACS Appl Mater Interfaces, 2016, 8: 13840-13848

23 Wang J, Zou M, Sun L, et al. Microfluidic generation of Buddha 
beads-like microcarriers for cell culture. Sci China Mater, 2017, 60: 857-865

24 Wang J, Shang L, Cheng Y, et al. Microfluidic generation of porous particles encapsulating spongy graphene for oil absorption. Small, 2015, 11: 3890-3895

25 Chen K, Li C, Shi L, et al. Growing three-dimensional biomorphic graphene powders using naturally abundant diatomite templates towards high solution processability. Nat Commun, 2016, 7: 13440

26 Sun J, Chen Z, Yuan L, et al. Direct chemical-vapor-depositionfabricated, large-scale graphene glass with high carrier mobility and uniformity for touch panel applications. ACS Nano, 2016, 10: 11136-11144

27 Wang J, Sun L, Zou M, et al. Bioinspired shape-memory graphene film with tunable wettability. Sci Adv, 2017, 3: e1700004

28 Cheng C, Wang D. Hydrogel-assisted transfer of graphene oxides into nonpolar organic media for oil decontamination. Angew Chem Int Ed, 2016, 55: 6853-6857

29 Ma C, Le X, Tang X, et al. A multiresponsive anisotropic hydrogel with macroscopic 3D complex deformations. Adv Funct Mater, 2016, 26: 8670-8676

30 Shang L, Wang Y, Yu Y, et al. Bio-inspired stimuli-responsive graphene oxide fibers from microfluidics. J Mater Chem A, 2017, 5: 15026-15030

31 Yang Z, Ren J, Zhang Z, et al. Recent advancement of nanostructured carbon for energy applications. Chem Rev, 2015, 115: 5159-5223

32 Li W, Wang J, Ren J, et al. 3D graphene oxide-polymer hydrogel: near-infrared light-triggered active scaffold for reversible cell capture and on-demand release. Adv Mater, 2013, 25: 6737-6743

$33 \mathrm{Ku} \mathrm{SH}$, Lee M, Park CB. Carbon-based nanomaterials for tissue engineering. Adv Healthcare Mater, 2013, 2: 244-260

34 Shi X, Chang H, Chen S, et al. Regulating cellular behavior on fewlayer reduced graphene oxide films with well-controlled reduction states. Adv Funct Mater, 2012, 22: 751-759

35 Cheng C, Li S, Thomas A, et al. Functional graphene nanomaterials based architectures: biointeractions, fabrications, and emerging biological applications. Chem Rev, 2017, 117: 1826-1914 36 Thompson BC, Murray E, Wallace GG. Graphite oxide to graphene. biomaterials to bionics. Adv Mater, 2015, 27: 7563-7582

37 Li Y, Lu Q, Liu H, et al. Antibody-modified reduced graphene oxide films with extreme sensitivity to circulating tumor cells. Adv Mater, 2015, 27: 6848-6854

38 Nichol JW, Koshy ST, Bae H, et al. Cell-laden microengineered gelatin methacrylate hydrogels. Biomaterials, 2010, 31: 5536-5544

39 Cui H, Zhang P, Wang W, et al. Near-infrared (NIR) controlled reversible cell adhesion on a responsive nano-biointerface. Nano Res, 2017, 10: 1345-1355

Acknowledgements This work was supported by the National Natural Science Foundation of China (21473029 and 51522302), the NSAF Foundation of China (U1530260), the Scientific Research Foundation of Southeast University, the Scientific Research Foundation of Graduate School of Southeast University, and the Postgraduate Research \& Practice Innovation Program of Jiangsu Province and the Fundamental Research Funds for the Central Universities.

Author contributions Wang J carried out the materials preparation and in vitro experiments; Chen $\mathrm{G}$ carried out the in vivo experiments; Zhao Y conceived the idea and designed the experiment; Wang J and Zhao Y analyzed data and wrote the paper; all authors contributed to general discussion of the article.

Conflict of interest The authors declare no conflict of interest.

Supplementary information Supporting data are available in the online version of the paper. 


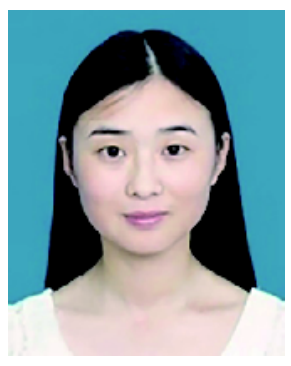

Jie Wang received her BSc degree from Southeast University in 2014. She is now a $\mathrm{PhD}$ candidate under the supervision of Prof. Yuanjin Zhao at Southeast University. Her research interest is the fabrication of functional materials based on microfluidics.

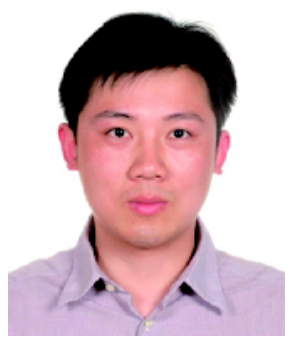

Yuanjin Zhao received his PhD degree in 2011 from Southeast University. In 2009-2010, he worked as a research scholar at Prof. David A. Weitz's group in SEAS of Harvard University. Since 2015, he was promoted to be a full professor of Southeast University. His current scientific interests include microfluidic-based materials fabrication, biosensors, and bioinspired photonic nanomaterials.

\section{智能响应性氧化石墨烯水凝胶微载体用于细胞的捕获与释放}

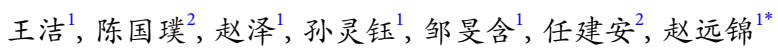

摘要 细胞微载体作为生物医学领域中三维细胞培养的一个平台, 通常会由于过于简单的材料组成成分, 使得微载体的功能局限于细胞 捕获与增殖. 本研究开发了一种近红外光响应的氧化石墨烯水凝胶微载体用于细胞的可控培养. 利用毛细管微流控技术, 通过乳化氧化石 墨烯一异丙基丙烯酰胺一甲基化明胶复合预聚溶液来制备智能响应性氧化石墨烯水凝胶微载体. 氧化石墨烯对近红外光辐射的吸收能力, 异丙基丙烯酰胺的热响应性相转换能力, 以及甲基化明胶良好的生物相容性, 使得该复合水凝胶微载体具有高效的光热响应的细胞捕 获、增殖以及释放能力. 此外, 近红外光响应的氧化石墨烯水凝胶微载体还能够为其内部包裹的细胞提供保护, 使其免受免疫系统的攻 击, 从而促进免疫活性小鼠体内肿瘤模型的形成与生长. 